\title{
Elastic-Plastic Invariant of Localized Deformation Autowaves
}

\author{
Nikita Ploskov ${ }^{1}$, Vladimir Danilov ${ }^{1,2, a)}$, and Lev Zuev ${ }^{1}$ \\ ${ }^{1}$ Institute of Strength Physics and Materials Science SB RAS, Tomsk, 634055, Russia \\ ${ }^{2}$ National Research Tomsk Polytechnic University, Tomsk, 634050, Russia \\ a) Corresponding author: dvi@ispms.tsc.ru
}

\begin{abstract}
The parameters of localization patterns emerging at the linear stage of work hardening are discussed in the frame of autowave concept. The stress-strain diagrams have been plotted for the test samples of metals exposed to active loading. It is found that the product of phase autowave velocity and length has the same order of magnitude as the product of elastic wave rate and the lattice constant of the respective metal; hence, this quantity can be regarded as elastic-plastic invariant. The invariant is found to vary in a regular manner with the shear modulus, modulus of dilatation and interplanar spacing. The data obtained for elements of one and the same period of Mendeleev's table of elements are found to vary monotonically; however, after changing over to the next period, a jump-wise variation in the data would occur. It can thus be concluded that the elastic-plastic invariant is a dominant characteristic of plasticity; the nature of the invariant is related to the lattice properties and the phonon density of crystal lattice.
\end{abstract}

Keywords: plastic deformation, localization, elasticity, self-organization, localization autowaves

\section{INTRODUCTION}

The plastic deformation in solids has been extensively studied since the 1990s. The main findings of the studies indicate that at micro- [1-3], meso- $[4,5]$ and macroscopic [6] scale levels the plastic flow would exhibit the inhomogeneous behavior from a yield limit to a material failure. According to authors of [6-8], the macrolocalization of plastic deformation is a characteristic feature observed for the deforming solid, no matter what loading conditions, crystal lattice type, phase composition and grain size of material are. In the course of macrolocalization development the localized plasticity autowaves were found to form.

The type of autowave emerging at the given flow stage in a deforming solid is determined by the plastic flow law, i.e. the multi-stage flow curve. Thus, at the yield plateau a switching autowave is observed; at the linear stage of work hardening, a phase autowave; at the parabolic (Taylor's) stage of work hardening, a stationary dissipative structure, and at the pre-fracture stage the onset of necking occurs, which is a forerunner of the imminent fracture of a material [6].

New standards of rigidity and consistency have been established in defining the characteristics of localized plastic flow autowaves; in particular, the autowave length, period and propagation rate have been defined experimentally for the linear stage of hardening. The phenomenon of localized plasticity autowaves was considered in the frame of synergetic approach. As a result, a significant relation, so-called 'elastic-plastic invariant', was established by L.B. Zuev [6, 7]. The elastic-plastic invariant is a product of the phase autowave rate and the phase autowave length. The values that comprise this relation have the same order of magnitude; hence, the invariant is good for all studied materials (see Table 1). For the same reason, the invariant is identical to the product of the rate of transverse elastic wave and the interplanar distance between the most closely packed planes of a respective metal, i.e.

$$
V_{\mathrm{aw}} \lambda=\varsigma V_{\perp} d,
$$


TABLE 1. Elastic-plastic invariants and interplanar distances of studied metals

\begin{tabular}{|c|c|c|c|c|c|c|c|c|}
\hline & Period & $\underset{\mathrm{nm}}{d,}$ & $\begin{array}{c}V \perp \cdot d \cdot 10^{7} \\
\mathrm{~m}^{2} / \mathrm{s}\end{array}$ & $\begin{array}{c}V_{\text {aw }} \cdot \lambda \cdot 10^{7} \\
\mathrm{~m}^{2} / \mathrm{s}\end{array}$ & $\begin{array}{c}G, \\
\text { GPa }\end{array}$ & $\begin{array}{c}K, \\
\text { GPa }\end{array}$ & $\begin{array}{c}\Gamma \cdot 10^{-7}, \\
\mathrm{~m}^{2} / \mathrm{s}\end{array}$ & $\begin{array}{c}\gamma \cdot 10^{-37} \\
\mathrm{~kg}^{-1} \mathrm{~m}^{-2} \mathrm{~s}\end{array}$ \\
\hline $\mathrm{Ti}$ & 4 & 0.2241 & 7.9 & 3.50 & 43.3 & 107.2 & 0.280 & 3.51 \\
\hline $\mathrm{V}$ & 4 & 0.2138 & 6.2 & 2.80 & 47.4 & 155.1 & 0.360 & 4.25 \\
\hline $\mathrm{Fe}$ & 4 & 0.2027 & 6.5 & 2.25 & 86.0 & 145.6 & 0.440 & 4.73 \\
\hline $\mathrm{Ni}$ & 4 & 0.2032 & 6.0 & 2.10 & 90.6 & 180.8 & 0.480 & 4.90 \\
\hline $\mathrm{Cu}$ & 4 & 0.2087 & 4,7 & 3.60 & 47.9 & 139.3 & 0.275 & 2.63 \\
\hline $\mathrm{Zn}$ & 4 & 0.2077 & 5.3 & 3.70 & 46.2 & 70.5 & 0.270 & 2.50 \\
\hline $\mathrm{Zr}$ & 5 & 0.2459 & 6.1 & 1.90 & 24.9 & 89.3 & 0.520 & 3.44 \\
\hline $\mathrm{Nb}$ & 5 & 0.2329 & 5.3 & 1.80 & 43.4 & 153.3 & 0.550 & 3.56 \\
\hline In & 5 & 0.2715 & 2.2 & 2.60 & 7.3 & 46.2 & 0.380 & 1.99 \\
\hline $\mathrm{Sn}$ & 5 & 0.2910 & 5.3 & 3.40 & 17.9 & 60.6 & 0.290 & 1.48 \\
\hline $\mathrm{Pb}$ & 6 & 0.2852 & 2.0 & 3.20 & 10.1 & 41.7 & 0.310 & 0.90 \\
\hline
\end{tabular}

where $0.3 \leq \varsigma \leq 1.6$. At present, we know enough to tackle some relevant questions, in particular, what is the essence of the elastic-plastic invariant and in what way it is related to the physical and chemical characteristics of metals? Moreover, the question about what basic characteristics enter the invariant remains open as yet.

\section{MAIN CONCEPTS, EXPERIMENTAL DATA AND DISCUSSION}

The autowave nature of plastic deformation localization was considered in the frame of two-component model of self-organizing plastic flow by L.B. Zuev [8]. The active medium, i.e. deforming solid, will separate into two controlling subsystems, i.e. dynamic and information ones. The former system is represented by mobile dislocations and ensembles thereof, which are responsible for the proper change of the form, and the latter subsystem, by acoustic emission signals emitted by the elementary relaxation acts. According to Kadomtsev [9], the interaction of these two subsystems will cause self-organization of the medium; as a result, different types of autowaves are liable to form. In the frame of this conceptual representation, plasticity and elasticity are given by the left and right sides of Eq. (1), respectively. Hence, the product of the autowave characteristics, $V_{\text {aw }} \lambda$, depends on the basic characteristics of the deforming solid, i.e. lattice parameters and elasticity modulus.

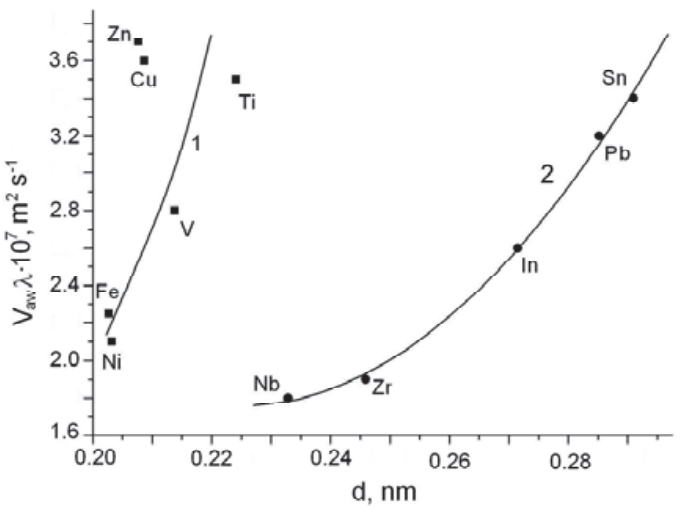

(a)

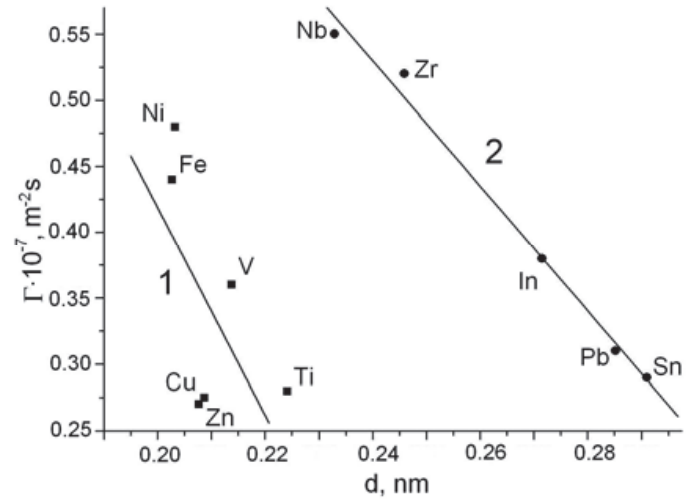

(b)

FIGURE 1. Dependencies of elastic-plastic invariant on the interplanar distance of the crystal lattice in metals of the fourth ( 1 ) and fifth (2) periods of Mendeleev's table of elements 


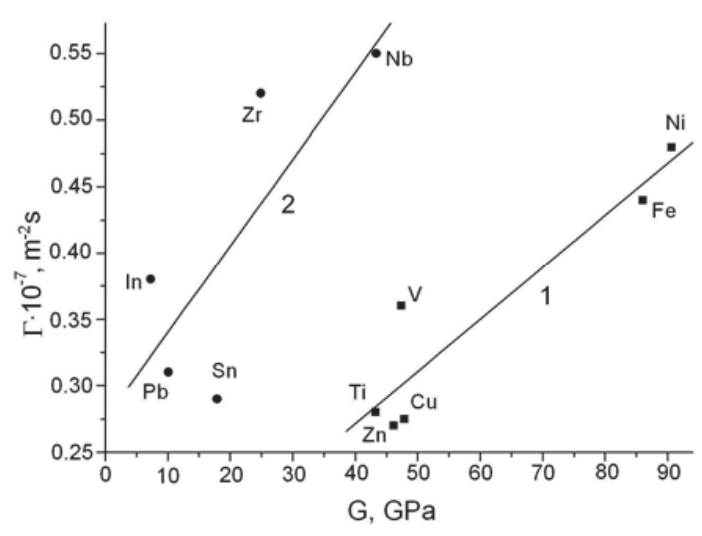

(a)

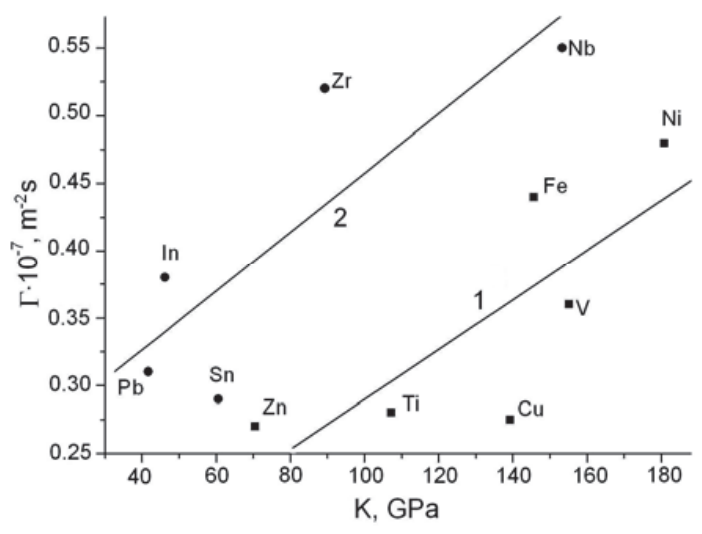

(b)

FIGURE 2. The inverse values of elastic-plastic invariant as a function of a) shear modulus and b) modulus of dilatation (b); metals of the fourth and fifth periods (lines 1 and 2, respectively)

Figure 1(a) presents the invariant $V_{\text {aw }} \lambda$ as a function of interplanar distance. It can be seen that the elements of the fourth and fifth periods fall into two different groups (see Table 1) with the exception of $\mathrm{Pb}$, which belongs to the sixth period, but neatly fits curve No. 2 in Fig. 1(a).

Both dependencies are non-linear; however, these are amenable by a linearization, provided $\Gamma=\left(V_{\mathrm{aw}} \lambda\right)^{-1}$ is plotted vs interplanar distance $d$. In so doing, the elements are grouped by periods more neatly. As seen from dependencies (1) and (2) in Fig. 1(b), the value $\Gamma$ decreases practically linearly in both groups with growing $d$.

Figure 2 presents the value $\Gamma$ as a function of shear modulus (a) and uniform dilatation (b). In this case, separation of elements also occurs according to their positions in Mendeleyev's table.

The fact that the dependencies $\Gamma(G), \Gamma(K)$ and $\Gamma(d)$ obtained for both groups of elements have practically the same inclination is of particular importance; hence, the elastic-plastic invariant has practically the same values on going from one period of Mendeleyev's table of elements to the other. In this respect, the invariant behavior is similar to that of the main chemical properties and of certain physical properties. Thus the atomic radius and the atomic volume [10] will decrease in mid-period, while the same values will increase in a jump-wise manner on changing over to the next period [11]. Similar dependencies are obtained for density, compressibility, melting temperature, heat and electrical conductivity as well as elasticity moduli.

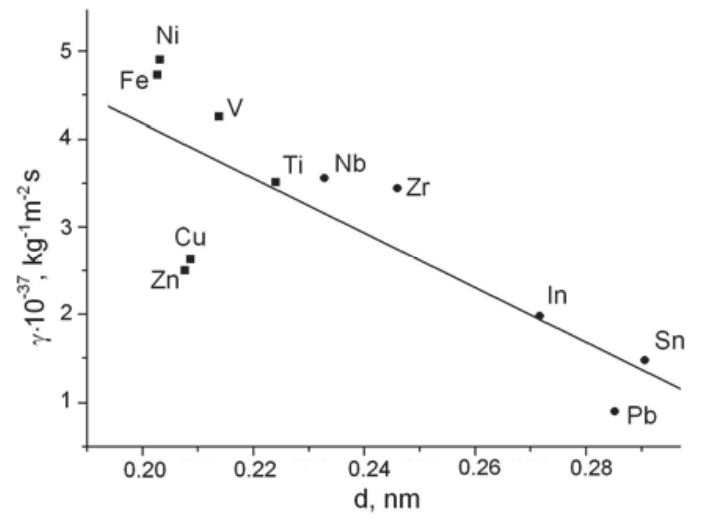

(a)

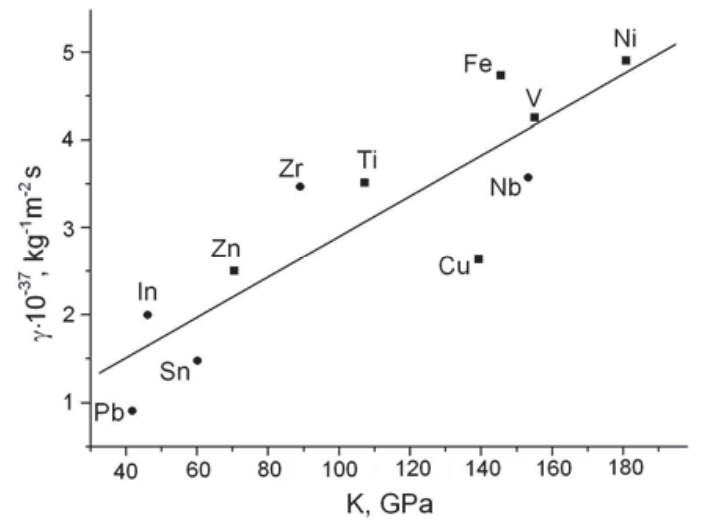

(b)

FIGURE 3. Normalized values of the elastic-plastic invariant vs interplanar distance (a) and uniform compression modulus (b); - - metals of the fourth period; $\bullet$ - metals of the fifth period 
Elements from one and the same group might fall into different periods. In this case, the elements will have different atomic masses and similar properties. Hence, the effect of atomic mass can be minimized by normalization of the quantity $\Gamma$ with respect to the value $A$ as

$$
\gamma=\frac{\Gamma}{A}=\frac{1}{\lambda V_{\mathrm{aw}} A}
$$

Indeed, in this case, the value $\gamma$ will decrease linearly with the growing interplanar distance in all studied elements (Fig. 3(a)); the same value will increase linearly with growing uniform compression modulus (Fig. 3(b)) or shear modulus.

Looking back at expression (1), we see that the products from the left and right sides can be regarded as invariants of plastic and elastic processes, respectively, which occur simultaneously in the deforming medium. Hence, small elastic strains $\varepsilon_{\mathrm{e}}<<1$ propagating at high rates $V_{\perp}$ control the redistribution of large plastic strains nuclei $\varepsilon_{\mathrm{p}} \approx 1$, which occurs at low rates, $V_{\text {aw }}$, and vice versa.

The above suggests that the phonon system plays an important role in the formation of localized plastic deformation autowaves. According to Taylor, such effect might be expected, since plastic strain increments occurring on the micro-scale level are due to the motion of dislocations overcoming local obstacles. In this situation the dislocations move over the local barriers; hence, their motion rate is affected by the phonon gas alone [12].

\section{CONCLUSION}

The interdependence of micro- and macro-scale deformation processes is expressed in terms of elastic-plastic invariant. The microscopic deformation defects will interact with the phonon subsystem of the deforming solid, forming thereby mobile macro-nuclei of localized plasticity, i.e. autowaves. Therefore, the elastic-plastic invariant is related to the lattice properties of crystals and to the density of phonon gas. The invariant will change according to the periodic law, i.e. the number of the given element in Mendeleev's table. It is thus concluded that the elasticplastic invariant is a basic characteristic of plastic deformation, just like the elastic modulus, the heat conductivity and the melting heat are the characteristics of thermal processes, while the ionization potential and the electric conductivity characterize electric processes.

\section{REFERENCES}

1. D. Kuhlmann-Wilsdorf, in Dislocations in Solids, edited by F. R. N. Nabarro and M. S. Duesbery (Elsevier, Amsterdam, Boston, 2002), p. 213.

2. G. A. Malygin, Physics-Uspekhi 42, 887 (1999).

3. N. Stanford, D. Dunne, and M. Ferry, Acta Mat. 51, 665 (2003).

4. P. Hähner and E. Rizzi, Acta Mat. 51, 3385 (2003).

5. V. E. Panin, V. E. Egorushkin, and A. V. Panin, Physics-Uspekhi 55, 1260 (2012).

6. L. B. Zuev, V. I. Danilov, and S. A. Barannikova, Plastic Flow Macrolocalization Physics (Nauka, Novosibirsk, 2008).

7. L. B. Zuev, V. I. Danilov, S. A. Barannikova, and V. V. Gorbatenko, Phys. Wave Phenom. 17, 66 (2009).

8. L. B. Zuev, Phys. Wave Phenom. 20, 166 (2012).

9. B. B. Kadomtsev, Dynamics and Information (Physics Uspekhi, Moscow, 1999).

10. L. Pauling, General Chemistry (W.H. Freeman \& Co., San-Francisco, 1970).

11. H. Gray, Electrons and Chemical Bonding (Benjamin, Inc., New York, Amsterdam, 1965).

12. V. I. Alshits and Yu. M. Sandler, Phys. Staus Solidi B 64, K45 (1974). 